\title{
APPLICATION OF THE LAPLACE-ADOMIAN METHOD AND THE SBA METHOD TO SOLVING THE PARTIAL DIFFERENTIAL AND INTEGRO- DIFFERENTIAL EQUATIONS
}

\section{BOUKARY BÉYI ${ }^{1}$, JOSEPH BONAZEBI-YINDOULA ${ }^{2}$, LONGIN SOME ${ }^{1}$ and GABRIEL BISSANGA ${ }^{2}$}

1 Université de Ouaga-1

Professeur Joseph KI ZERBO

Burkina Faso

e-mail: bonayindoula@yahoo.fr

2Université Marien NGOUABI Brazzaville

Congo

\begin{abstract}
In this paper, we use firstly the Laplace-Adomian method to construct the solution of a kind of Fischer's equation and secondly the SBA method to construct the solution of an integral-differential equation.
\end{abstract}

\section{Introduction}

Many problems in natural and engineering sciences are modelled by partial differential equations (PDEs) and integral equations. Most of these equations are nonlinear. Several techniques have been used to find

2020 Mathematics Subject Classification: 47H14, 34G20, 47J25, 65J15.

Keywords and phrases: SBA method, Laplace-Adomian method, Fischer's equation, Fredholm integral equation.

Received January 11, 2020; Revised February 21, 2020

(C) 2020 Scientific Advances Publishers 
the analytical solutions of such equations, like the perturbation method, the homotopy perturbation method, the Adomian decomposition method, the Laplace-Adomian method, the SBA method and others [3], [4], [19], [20]. Here we use the Laplace-Adomian method and the SBA method to investigate a kind of Fischer's equation and an Fredholm integrodifferential equation.

\subsection{About Laplace-Adomian method and the SBA method}

About Laplace-Adomian method, we can see [3]-[9]. Using the simple Laplace transformations, it is difficult to find the solution of nonlinear differential equation. But, the combination of the Laplace transformations and the Adomian decomposition method allows us to avoid this difficulty.

\subsubsection{The Laplace-Adomian decomposition method (LADM)}

Suppose that we need to solve the following equation:

$$
F(u(x, t))=h(x, t)
$$

with the following initial condition:

$$
u(x, 0)=f(x)
$$

in a Banach space $E$, where $F: E \rightarrow E$ is a linear or a nonlinear operator, $h \in E$ and $u$ is the unknown function. The principle of LADM decomposition which has been the subject of several studies [6], [7], [19] is based on the decomposition of the operator $F$ in the following form:

$$
F=L+R+N
$$

where $L+R$ is linear, $N$ is nonlinear, $L$ is invertible with $L^{-1}$ as inverse.

Using that decomposition, Equation (1) is equivalent to

$$
L u+R u+N u=h(x, t) .
$$

We denote $L=\frac{\partial(.)}{\partial t}$ and $L^{-1}=\int_{0}^{t}() d$.$s .$ 
Let's note the Laplace transform by $\mathcal{L}(L u(x, t))=\int_{0}^{\infty} u(x, t) e^{-s t} d t$.

From (4), we have

$$
\mathcal{L}(L u(x, t))+\mathcal{L}(R u(x, t))+\mathcal{L}(N u(x, t))=\mathcal{L}(h(x, t))
$$

$\Leftrightarrow$

$$
s \mathcal{L}(u(x, t))-u(x, 0)+\mathcal{L}(R u(x, t))+\mathcal{L}(N u(x, t))=\mathcal{L}(h(x, t))
$$

$\Leftrightarrow$

$$
s \mathcal{L}(u(x, t))=u(x, 0)+\mathcal{L}(h(x, t))-\mathcal{L}(R u(x, t))-\mathcal{L}(N u(x, t))
$$

Thus

$$
\begin{aligned}
& \mathcal{L}(u(x, t))=\frac{1}{s} u(x, 0)+\frac{1}{s} \mathcal{L}(h(x, t))-\frac{1}{s} \mathcal{L}(R u(x, t))-\frac{1}{s} \mathcal{L}(N u(x, t)) \\
\Leftrightarrow & \\
& \mathcal{L}(u(x, t))=\frac{1}{s} f(x)+\frac{1}{s} \mathcal{L}(h(x, t))-\frac{1}{s} \mathcal{L}(R u(x, t))-\frac{1}{s} \mathcal{L}(N u(x, t)) .
\end{aligned}
$$

We look for the solution of (1) in the following form:

$$
u(x, t)=\sum_{n=0}^{\infty} u_{n}(x, t)
$$

and suppose that

$$
N(u(x, t))=\sum_{n=0}^{\infty} A_{n}(x, t),
$$

where $A_{n}$ are special polynomials $u_{0}, u_{1}, u_{2}, \cdots, u_{n}$ called Adomian polynomials and defined by [5]-[8]:

$$
A_{n}=\frac{1}{n !}\left[\frac{d^{n}}{d \lambda^{n}} N\left(\sum_{i=0}^{+\infty} \lambda^{i} u_{i}\right)\right]_{\lambda=0}, n=0,1,2, \ldots
$$

where $\lambda$ is a parameter used by "convenience". 
We can also use the following formulas:

$$
\left\{\begin{array}{l}
A=N\left(\varphi_{0}\right) \\
A_{n+1}=\frac{1}{n+1} \sum_{\mathrm{k}=0}^{n}(k+1) \varphi_{k+1} \frac{\partial A_{n}}{\partial \varphi_{k}}
\end{array}\right.
$$

From (10) and (11), the Equation (9) becomes

$$
\sum_{n=0}^{\infty} \mathcal{L}\left(u_{n}(x, t)\right)=\frac{1}{s} f(x)+\frac{1}{s} \mathcal{L}(h(x, t))-\sum_{n=0}^{\infty}\left(\frac{1}{s} \mathcal{L}\left(R u_{n}(x, t)\right)+\frac{1}{s} \mathcal{L}\left(A_{n}(x, t)\right)\right) .
$$

According to the classic theory of the Adomian decomposition method [6]-[8], we construct the following Adomian algorithm:

$$
\left\{\begin{array}{l}
\mathcal{L}\left(u_{0}(x, t)\right)=\frac{1}{s} f(x)+\frac{1}{s} \mathcal{L}(h(x, t)) \\
\mathcal{L}\left(u_{n+1}(x, t)\right)=-\frac{1}{s} \mathcal{L}\left(R u_{n}(x, t)\right)-\frac{1}{s} \mathcal{L}\left(A_{n}(x, t)\right), n \geq 0
\end{array}\right.
$$

The inverse Laplace transform gives as

$$
\left\{\begin{array}{l}
u_{0}(x, t)=\mathcal{L}^{-1}\left(\frac{1}{s} f(x)+\frac{1}{s} \mathcal{L}(h(x, t))\right) \\
u_{n+1}(x, t)=\mathcal{L}^{-1}\left(-\frac{1}{s} \mathcal{L}\left(R u_{n}(x, t)\right)-\frac{1}{s} \mathcal{L}\left(A_{n}(x, t)\right), n \geq 0\right) .
\end{array}\right.
$$

\subsubsection{SBA method}

About SBA method, we can see [1], [2]. The SBA method is powerful than the Adomian decomposition method because the SBA method avoids us the calculation of the Adomian polynomials.

The SBA method is based on the Adomian decomposition method, the successive approximation method and the Picard principle. Suppose that we need to solve the following equation:

$$
A u=f,
$$


where $A: H \rightarrow H$, is a linear or nonlinear operator and $H$ is a Hilbert space. Let's suppose that we can decompose the operator $A$ in the following form:

$$
A=L+R+N
$$

where $L+R$ is the linear part and $N$ is the nonlinear part. We suppose that $L$ is invertible with $L^{-1}$ as inverse, then the Equation (17) becomes

$$
L u+R u+N u=f \Leftrightarrow u=\theta+L^{-1} f-L^{-1} R u-L^{-1} N u,
$$

where $\theta$ is such that $L \theta=0$. Equation (19) is the Adomian canonical form. Using the successive approximations, we get

$$
\left\{\begin{array}{l}
u^{k}=\theta^{k}+L^{-1} f^{k}-L^{-1} R\left(u^{k}\right)-L^{-1} N\left(u^{k-1}\right) ; k \geq 1, \\
\text { with } \\
f^{1}=f^{2}=\cdots=f ; \theta^{1}=\theta^{2}=\cdots=\theta .
\end{array}\right.
$$

We look for the solution of (17), for every $k \geq 1$ in the following form $u^{k}=\sum_{n=0}^{+\infty} u_{n}^{k}(x, t)$ and we suppose that series converges.

Therefore, $u=\lim _{k \rightarrow+\infty} u^{k}$ is the solution of the Equation (17).

(20) yields the following Adomian algorithm [2]-[12]:

$$
\left\{\begin{array}{l}
u_{0}^{k}=\theta^{k}+L^{-1} f^{k}-L^{-1} N\left(u^{k-1}\right) \\
u_{n+1}^{k}=-L^{-1} R\left(u_{n}^{k}\right), n \geq 0
\end{array} k \geq 1 .\right.
$$

According to the Picard principle, for every $k \geq 1$, one must choose $u^{k-1}$ as $N\left(u^{k-1}\right)=0$. Thus; 
For $k=1$, we have

$$
\left\{\begin{array}{l}
u_{0}^{1}=\theta+L^{-1} f \\
u_{n+1}^{1}=-L^{-1} R\left(u_{n}^{1}\right), n \geq 0
\end{array}\right.
$$

we suppose that the condition $N(u)=0$ is satisfied then $u^{1}=\sum_{n=0}^{+\infty} u_{n}^{1}(x, t)$.

For $k=2$, we have

$$
\left\{\begin{array}{l}
u_{0}^{2}=\theta+L^{-1} f \\
u_{n+1}^{2}=-L^{-1} R\left(u_{n}^{2}\right), n \geq 0 .
\end{array}\right.
$$

Here we must verify that $N\left(u^{1}\right)=0$ then $u^{2}=\sum_{n=0}^{+\infty} u_{n}^{2}(x, t)$.

We make the same procedure for $k \geq 2$ and the solution of Equation (17) is $u=\lim _{\mathrm{k} \rightarrow+\infty} u^{k}$.

\section{Application of the Laplace-Adomian Method to Solve a Kind of Fischer's Equation}

We consider the following initial value problem [17]:

$$
\left\{\begin{array}{l}
\frac{\partial u(x, t)}{\partial t}-\alpha \frac{\partial^{2} u(x, t)}{\partial x^{2}}=\varepsilon u(x, t)-\lambda u^{q+1}(x, t), \quad \forall t>0, \\
u(x, 0)=\beta,
\end{array}\right.
$$

where $\lambda, \alpha, \varepsilon \in \mathbb{R}$ and $q \in \mathbb{R}^{*}$.

Let's put

$$
N u(x, t)=u^{q+1}(x, t) .
$$


From (24), we have

$$
\frac{\partial u(x, t)}{\partial t}=u(x, t)+\alpha \frac{\partial^{2} u(x, t)}{\partial x^{2}}-\lambda u^{q+1}(x, t) .
$$

Let's note the Laplace transform by

$$
U(x, s)=\mathcal{L}(u(x, t))=\int_{0}^{\infty} u(x, t) e^{-s t} d t .
$$

From (27), we obtain

$$
\mathcal{L}\left(\frac{\partial u(x, t)}{\partial t}\right)=\int_{0}^{\infty} e^{-s t} \frac{\partial u}{\partial t} d t=s U(x, s)-u(x, 0) .
$$

Applying the Laplace transformations on (26), we have

$$
\mathcal{L}(u(x, t))=\frac{\beta}{s-\varepsilon}+\frac{\alpha}{s-\varepsilon} \mathcal{L}\left(\frac{\partial^{2} u(x, t)}{\partial x^{2}}\right)-\frac{\lambda}{s-\varepsilon} \mathcal{L}(N u(x, t)) .
$$

Using the Laplace inverse transformation, we obtain

$$
u(x, t)=\beta e^{\varepsilon t}+\alpha \mathcal{L}^{-1}\left(\frac{1}{s-\varepsilon} \mathcal{L}\left(\frac{\partial^{2} u(x, t)}{\partial x^{2}}\right)\right)-\lambda \mathcal{L}^{-1}\left(\frac{1}{s-\varepsilon} \mathcal{L}(N u(x, t))\right)
$$

According to the Adomian decomposition method, we look for the solution of (24) in the following form:

$$
u(x, t)=\sum_{n=0}^{+\infty} u_{n}(x, t)
$$

and we suppose that

$$
N u(x, t)=\sum_{n=0}^{+\infty} A_{n}(x, t)
$$

From [13], we get

$$
\left\{\begin{array}{l}
A_{0}(x, t)=N\left(u_{0}(x, t)\right), \\
A_{n+1}(x, t)=\frac{1}{n+1} \sum_{k=0}^{n}(k+1) u_{k+1} \frac{\partial A_{n}}{\partial u_{k}}, n \in \mathbb{N} .
\end{array}\right.
$$


Substituting (31) and (32) into (30), we obtain the following Adomian algorithm:

$$
\left\{\begin{array}{l}
u_{0}(x, t)=\beta e^{\varepsilon t} \\
u_{n+1}(x, t)=+\alpha \mathcal{L}^{-1}\left(\frac{1}{s-\varepsilon} \mathcal{L}\left(\frac{\partial^{2} u(x, t)}{\partial x^{2}}\right)\right)-\lambda \mathcal{L}^{-1}\left(\frac{1}{s-\varepsilon} \mathcal{L}\left(A_{n}(x, t)\right)\right) .
\end{array}\right.
$$

From (33), we obtain

$$
\left\{\begin{array}{l}
A_{0}=u_{0}^{q+1}, \\
A_{1}=(q+1)\left(\frac{u_{1}}{u_{0}}\right) u_{0}^{q+1} \\
A_{2}=\frac{(q+1)(2 q+1)}{2 !}\left(\frac{u_{1}}{u_{0}}\right)^{2} u_{0}^{q+1}, \\
A_{3}=\frac{(q+1)(2 q+1)(3 q+1)}{3 !}\left(\frac{u_{1}}{u_{0}}\right)^{3} u_{0}^{q+1}, \\
\quad \cdots \\
A_{n}=\frac{\prod_{k=0}^{n}(k q+1)}{n !}\left(\frac{u_{1}}{u_{0}}\right)^{n} u_{0}^{q+1} .
\end{array}\right.
$$

From (34), we obtain

$$
\left\{\begin{array}{l}
u_{0}(x, t)=\beta e^{\varepsilon t}, \\
u_{1}(x, t)=\left(-\frac{\lambda \beta^{q}}{\varepsilon q}\left(e^{q \varepsilon t}-1\right)\right) \beta e^{\varepsilon t}, \\
u_{2}(x, t)=\frac{q+1}{2 !}\left(-\frac{\lambda \beta^{q}}{\varepsilon q}\left(e^{q \varepsilon t}-1\right)\right)^{2} \beta e^{\varepsilon t}, \\
u_{3}(x, t)=\frac{(q+1)(2 q+1)}{3 !}\left(-\frac{\lambda \beta^{q}}{\varepsilon q}\left(e^{q \varepsilon t}-1\right)\right)^{3} \beta e^{\varepsilon t}, \\
u_{4}(x, t)=\frac{(q+1)(2 q+1)(3 q+1)}{4 !}\left(-\frac{\lambda \beta^{q}}{\varepsilon q}\left(e^{q \varepsilon t}-1\right)\right)^{4} \beta e^{\varepsilon t}, \\
u_{n}(x, t)=\frac{\prod_{k=0}^{n-1}(\mathrm{k} q+1)}{n !}\left(-\frac{\lambda \beta^{q}}{\varepsilon q}\left(e^{q \varepsilon t}-1\right)\right)^{n} \beta e^{\varepsilon t}, n \geq 1 .
\end{array}\right.
$$


So,

$$
u(x, t)=\left[1+\sum_{n=1}^{+\infty} \frac{\prod_{k=0}^{n-1}(k q+1)}{n !}\left(-\frac{\lambda \beta^{q}}{\varepsilon q}\left(e^{q \varepsilon t}-1\right)\right)^{n}\right] \beta e^{\varepsilon t}
$$

Lemma. According to the D'Alembert criteria, the following series

$$
\sum_{n=1}^{+\infty} \frac{\prod_{k=0}^{n-1}(k q+1)}{n !}\left(-\frac{\lambda \beta^{q}}{\varepsilon q}\left(e^{q \varepsilon t}-1\right)\right)^{n}
$$

is absolutely convergent for all $t \in\left[\frac{1}{q \varepsilon} \ln \left(1-\frac{q \varepsilon}{\lambda(|q|+1) \beta^{q}}\right), \frac{1}{q \varepsilon} \ln \right.$ $\left.\left(1+\frac{q \varepsilon}{\lambda(|q|+1) \beta^{q}}\right)\right]$ and the remain term $R_{n}$ verifies the following relation:

$$
0 \leq R_{n} \leq \frac{\left|-\frac{\lambda \beta^{q}}{\varepsilon}\left(e^{q \varepsilon t}-1\right)\right|}{1-\left|-\frac{\lambda \beta^{q}}{\varepsilon}\left(e^{q \varepsilon t}-1\right)\right|}
$$

Proof. Let's denote

$$
a_{n}=\frac{\prod_{k=0}^{n-1}(k q+1)}{n !}
$$

and

$$
b_{n}=\left(-\frac{\lambda \beta^{q}}{\varepsilon q}\left(e^{q \varepsilon t}-1\right)\right)^{n} .
$$


We remark that

$$
\begin{gathered}
\left|\frac{a_{n+1}}{a_{n}}\right| \leq\left|\frac{n q+1}{n+1}\right| \leq\left|\frac{n q}{n+1}\right|+\left|\frac{1}{n+1}\right| \leq|q|+1, \\
\left|\frac{b_{n+1}}{b_{n}}\right|=\left|-\frac{\lambda \beta^{q}}{\varepsilon q}\left(e^{q \varepsilon t}-1\right)\right| .
\end{gathered}
$$

From (42) and (43), we have

$$
\begin{aligned}
\left|\frac{a_{n+1}}{a_{n}} \frac{b_{n+1}}{b_{n}}\right|=\left|\frac{a_{n+1}}{a_{n}}\right|\left|\frac{b_{n+1}}{b_{n}}\right| \leq 1 & \Leftrightarrow(|q|+1)\left|-\frac{\lambda \beta^{q}}{\varepsilon q}\left(e^{q \varepsilon t}-1\right)\right| \leq 1 \\
& \Leftrightarrow\left|-\frac{\lambda \beta^{q}(|q|+1)}{\varepsilon q}\left(e^{q \varepsilon t}-1\right)\right| \leq 1 \\
& \Leftrightarrow-1 \leq-\frac{\lambda \beta^{q}(|q|+1)}{\varepsilon q}\left(e^{q \varepsilon t}-1\right) \leq 1 \\
& \Leftrightarrow-\frac{\varepsilon q}{\lambda(|q|+1) \beta^{q}} \leq e^{q \varepsilon t}-1 \leq \frac{\varepsilon q}{\lambda(|q|+1) \beta^{q}} \\
& \Leftrightarrow 1-\frac{\varepsilon q}{\lambda(|q|+1) \beta^{q}} \leq e^{q \varepsilon t} \leq 1+\frac{\varepsilon q}{\lambda(|q|+1) \beta^{q}} .
\end{aligned}
$$

If $\lambda(|q|+1) \beta^{q}>\varepsilon q$, we get

$$
\frac{1}{q \varepsilon} \ln \left(1-\frac{\varepsilon q}{\lambda(|q|+1) \beta^{q}}\right) \leq t \leq \frac{1}{q \varepsilon} \ln \left(1+\frac{\varepsilon q}{\lambda(|q|+1) \beta^{q}}\right) .
$$

Proposition. Suppose that $(q, m) \in \mathbb{R}^{*} \times \mathbb{N}^{*},(\beta, \lambda, \varepsilon) \in \mathbb{R}^{3}, x \in \mathbb{R}^{m}$, $\alpha \in \mathbb{R}^{m}, \delta \in \mathbb{R}^{m}, \Omega=\left[\frac{1}{q \varepsilon} \ln \left(1-\frac{q \varepsilon}{\lambda(|q|+1) \beta^{q}}\right), \frac{1}{q \varepsilon} \ln \left(1+\frac{q \varepsilon}{\lambda(|q|+1) \beta^{q}}\right)\right]$, then, 
the solution of the following problem:

$$
\begin{gathered}
\frac{\partial u(x, t)}{\partial t}-\nabla^{k} u(x, t) . \alpha+\nabla u(x, t) . \delta=\varepsilon u(x, t)-\lambda u^{q+1}(x, t), k \geq 2, \\
u(x, 0)=\beta
\end{gathered}
$$

on the set $\mathbb{R}^{m} \times \Omega$ is

$$
u(x, t)=\left[1+\sum_{n=1}^{+\infty} \frac{\prod_{k=0}^{n-1}(k q+1)}{n !}\left(-\frac{\lambda \beta^{q}}{\varepsilon q}\left(e^{q \varepsilon t}-1\right)\right)^{n}\right] \beta e^{\varepsilon t},
$$

where $u . \nu$ is the scalar product of $u$ and $\nu$. Otherwise, this series solution is convergent on $\mathbb{R}^{m} \times \Omega$.

Proof. If $t=0$, from (48), we obtain (46).

If $t \neq 0$, from (48), we get

$$
\begin{aligned}
\frac{\partial u(x, t)}{\partial t}= & u_{0}(x, t) \frac{d}{d t}\left[1+\sum_{n=1}^{+\infty} \frac{\prod_{k=0}^{n-1}(k q+1)}{n !}\left(-\frac{\lambda \beta^{q}}{\varepsilon q}\left(e^{q \varepsilon t}-1\right)\right)^{n}\right] \\
& +\left[1+\sum_{n=1}^{+\infty} \frac{\prod_{k=0}^{n-1}(k q+1)}{n !}\left(-\frac{\lambda \beta^{q}}{\varepsilon q}\left(e^{q \varepsilon t}-1\right)\right)^{n}\right] \frac{\partial u_{0}(x, t)}{\partial t} .
\end{aligned}
$$

We firstly remark that

$$
\frac{\partial u_{0}(x, t)}{\partial t}=\varepsilon u_{0}(x, t) \text { and }\left[1+\sum_{n=1}^{+\infty} \frac{\prod_{k=0}^{n-1}(k q+1)}{n !}\left(-\frac{\lambda \beta^{q}}{\varepsilon q}\left(e^{q \varepsilon t}-1\right)\right)^{n}\right]=\frac{u(x, t)}{u_{0}(x, t)}
$$


so

$$
\left[1+\sum_{n=1}^{+\infty} \frac{\prod_{k=0}^{n-1}(k q+1)}{n !}\left(-\frac{\lambda \beta^{q}}{\varepsilon q}\left(e^{q \varepsilon t}-1\right)\right)^{n}\right] \frac{\partial u_{0}(x, t)}{\partial t}=\varepsilon u(x, t)
$$

on the other hand,

$$
\begin{aligned}
& u_{0}(x, t) \frac{d}{d t}\left[1+\sum_{n=1}^{+\infty} \frac{\prod_{k=0}^{n-1}(k q+1)}{n !}\left(-\frac{\lambda \beta^{q}}{\varepsilon q}\left(e^{q \varepsilon t}-1\right)\right)^{n}\right]=-\lambda u_{0}^{q+1}(x, t) \sum_{n=1}^{+\infty} \frac{\prod_{k=0}^{n-1}(k q+1)}{(n-1) !} \\
& \times\left(-\frac{\lambda \beta^{q}}{\varepsilon q}\left(e^{q \varepsilon t}-1\right)\right)^{n-1} \\
&=-\lambda u_{0}^{q+1}(x, t) \sum_{N=0}^{+\infty} \frac{\prod_{k=0}^{N}(k q+1)}{N !}\left(-\frac{\lambda \beta^{q}}{\varepsilon q}\left(e^{q \varepsilon t}-1\right)\right)^{N} \cdot
\end{aligned}
$$

Secondly:

$$
\sum_{N=0}^{+\infty} \frac{\prod_{k=0}^{N}(k q+1)}{N !}\left(-\frac{\lambda \beta^{q}}{\varepsilon q}\left(e^{q \varepsilon t}-1\right)\right)^{N}=\sum_{N=0}^{+\infty} \frac{A_{N}}{u_{0}^{q+1}(x, t)},
$$


so

$$
\begin{aligned}
u_{0}(x, t) \frac{d}{d t}\left[1+\sum_{n=1}^{+\infty} \frac{\prod_{k=0}^{n-1}(k q+1)}{n !}\left(-\frac{\lambda \beta^{q}}{\varepsilon q}\left(e^{q \varepsilon t}-1\right)\right)^{n}\right] & =-\lambda u_{0}^{q+1}(x, t) \sum_{n=0}^{+\infty} \frac{A_{N}}{u_{0}^{q+1}(x, t)} \\
& =-\lambda \sum_{N=0}^{+\infty} A_{N} \\
& =-\lambda N u(x, t) \\
& =-\lambda u^{q+1}(x, t)
\end{aligned}
$$

Thirdly, $u(x, t)$ depends only on $t$ variable, so

$$
\nabla^{k} u(x, t) \cdot \alpha+\nabla u(x, t) . \delta=0
$$

Finally,

$$
\frac{\partial u(x, t)}{\partial t}-\nabla^{k} u(x, t) . \alpha+\nabla u(x, t) . \delta=\varepsilon u(x, t)-\lambda u^{q+1}(x, t) .
$$

Remark. If $q=1, \varepsilon=1, \lambda=1, \alpha=1, \delta=1$ and $m=1$, we recover the case examined in [17], and we have

$$
u(x, t)=\frac{\beta e^{t}}{1-\beta+\beta e^{t}}
$$




\section{Application of the SBA Method to Solve a Fredholm Integro-Differential Equation}

Let's consider the following Cauchy problem [18]:

$$
\left\{\begin{aligned}
\frac{\partial u(x, t)}{\partial t}= & u(x, t)+\int_{0}^{1} t u^{\alpha}(x, t) d t-\frac{f^{\alpha}(x)}{\alpha^{2}}\left((\alpha-1) e^{\alpha}+1\right),(x, t) \in \mathbb{R} \times \mathbb{R}_{+}^{*}, \\
& u(x, 0)=f(x) .
\end{aligned}\right.
$$

We are going to construct the solution of this problem using the SBA method, where $f \in \mathbb{C}^{\infty}(\mathbb{R}), \alpha \in \mathbb{R}^{*}$.

Let's note $N(u(x, t))=\int_{0}^{1} t u^{\alpha}(x, t) d t-\frac{f^{\alpha}(x)}{\alpha^{2}}\left((\alpha-1) e^{\alpha}+1\right)$. From (57), we have

$u(t, x)=u(x, 0)+\int_{0}^{t} u(s, x) d s+\int_{0}^{t}\left[\int_{0}^{t} t u^{\alpha}(t, x) d t-\frac{f^{\alpha}(x)}{\alpha^{2}}\left((\alpha-1) e^{\alpha}+1\right)\right] d s$.

(58) is equivalent to

$$
u(x, t)=u(x, 0)+\int_{0}^{t} u(s, x) d s+\int_{0}^{t} N(u(x, s)) d s
$$

According to the SBA method, we suppose that the solution of (57) has the following form:

$$
u(x, t)=\lim _{\mathrm{k} \rightarrow+\infty} u^{k}(x, t)
$$

where

$$
u^{k}(x, t)=\sum_{n=0}^{+\infty} u_{n}^{k}(x, t) ; k \geq 1
$$


and for every $k \geq 1$, we get $u_{n}^{k}(x, t)$ for $n \geq 0$ through the following SBA algorithm:

$$
\left\{\begin{array}{l}
u_{0}^{k}(x, t)=u^{k}(x, 0)+\int_{0}^{t} N u^{k-1}(x, s) d s ; k \geq 1, \\
u_{n+1}^{k}(x, t)=\int_{0}^{t} u(s, x) d s ; n \geq 0 .
\end{array}\right.
$$

Here, $u^{k}(x, 0)=u(x, 0)=f(x)$.

The SBA principle needs that, for $k=1$, we must choose $u^{0}(x, t)$ like $N u^{0}(x, t)=0$ and for $k>1$, we must verify that $N u^{k-1}(x, t)=0$.

Thus; for $k=1$, we have the following SBA algorithm:

$$
\left\{\begin{array}{l}
u_{0}^{1}(x, t)=f(x)+\int_{0}^{t} N u^{0}(x, s) d s, \\
u_{n+1}^{1}(x, t)=\int_{0}^{t} u_{n}^{1}(x, s) d s ; n \geq 0 .
\end{array}\right.
$$

Here, taking $u^{0}(x, t)=e^{t} f(x)$, we obtain $N u^{0}(x, t)=0$, and from (63) we have the following algorithm:

$$
\left\{\begin{array}{l}
u_{0}^{1}(x, t)=f(x) \\
u_{n+1}^{1}(x, t)=\int_{0}^{t} u_{n}^{1}(x, s) d s ; n \geq 0 .
\end{array}\right.
$$


From (64), we obtain

$$
\left\{\begin{array}{l}
u_{1}^{1}(x, t)=t f(x), \\
u_{2}^{1}(x, t)=\frac{t^{2}}{2 !} f(x), \\
u_{3}^{1}(x, t)=\frac{t^{3}}{3 !} f(x), \\
\cdots \\
u_{n}^{1}(x, t)=\frac{t^{n}}{n !} f(x) \frac{t^{n}}{n !} f(x) .
\end{array}\right.
$$

From (65), we obtain

$$
u^{1}(x, t)=\sum_{n=0}^{+\infty} u_{n}^{1}(x, t)=\sum_{n=0}^{+\infty} \frac{t^{n}}{n !} f(x)=f(x) \sum_{n=0}^{+\infty} \frac{t^{n}}{n !}=e^{t} f(x)
$$

We note that $u^{1}(x, t)=u^{0}(x, t)=e^{t} f(x)$.

So; for $k=2$, we have $N u^{1}(x, t)=N u^{0}(x, t)=0$, therefore we have the same algorithm like for $k=1$, then

$$
\left\{\begin{array}{l}
u_{0}^{2}(x, t)=f(x), \\
u_{n+1}^{2}(x, t)=\int_{0}^{t} u_{n}^{1}(x, s) d s ; n \geq 0,
\end{array}\right.
$$

and we obtain

$$
u^{2}(x, t)=e^{t} f(x)
$$

Using the same procedure, for $k \geq 3$, we have $u^{k}(x, t)=e^{t} f(x)$.

So,

$$
u(x, t)=\lim _{k \rightarrow+\infty} u^{k}(x, t)=\lim _{k \rightarrow+\infty} e^{t} f(x)=e^{t} f(x)
$$


Proposition. The solution of the following Cauchy problem:

$\left\{\begin{array}{l}\frac{\partial u(x, t)}{\partial t}=u(x, t)+\int_{0}^{1} t u^{\alpha}(x, t) d t-\frac{f^{\alpha}(x)}{\alpha^{2}}\left((\alpha-1) e^{\alpha}+1\right),(x, t) \in \mathbb{R}^{m} \times \mathbb{R}_{+}^{*}, \\ u(x, 0)=f(x),\end{array}\right.$

where $f \in \mathbb{C}^{\infty}\left(\mathbb{R}^{m}\right), m \in \mathbb{N}^{*}$ and $\alpha \in \mathbb{R}^{*}$, is

$$
u\left(x_{1}, x_{2}, \ldots, x_{m}, t\right)=f\left(x_{1}, x_{2}, \ldots, x_{m}, t\right) e^{t}
$$

Proof. If $t=0$, we have $u\left(x_{1}, x_{2}, \ldots, x_{m}, 0\right)=f\left(x_{1}, x_{2}, \ldots, x_{m}\right) e^{0}$ $=f\left(x_{1}, x_{2}, \ldots, x_{m}\right)$.

If $t \neq 0$, we have

$$
\left\{\begin{aligned}
\int_{0}^{1} t u^{\alpha}(x, t) d t & =\int_{0}^{1} t f^{\alpha}(x) e^{\alpha t} d t \\
& =f^{\alpha}(x) \int_{0}^{1} t e^{\alpha t} d t \\
& =f^{\alpha}(x)\left(\frac{e^{\alpha}}{\alpha}-\frac{1}{\alpha} \int_{0}^{1} t e^{\alpha t} d t\right) \\
& =f^{\alpha}(x)\left[\frac{e^{\alpha}}{\alpha}-\frac{1}{\alpha^{2}}\left(e^{\alpha}-1\right)\right] \\
& =f^{\alpha}(x) \frac{(\alpha-1) e^{\alpha}+1}{\alpha^{2}},
\end{aligned}\right.
$$

and

$$
\frac{\partial u\left(x_{1}, x_{2}, \ldots, x_{m}, t\right)}{\partial t}=f\left(x_{1}, x_{2}, \ldots, x_{m}, t\right) e^{t}=u\left(x_{1}, x_{2}, \ldots, x_{m}, t\right)
$$

So

$$
\frac{\partial u(x, t)}{\partial t}=u(x, t)+\int_{0}^{1} t u^{\alpha}(x, t) d t-\frac{f^{\alpha}(x)}{\alpha^{2}}\left((\alpha-1) e^{\alpha}+1\right) .
$$




\section{Conclusion}

Through these two examples, we showed on the one hand the superiority of the Laplace-Adomian method in relation to the Laplace transformations and on the other hand the efficiency of the SBA method in the resolution of the ordinary and partial differential equations, and integro-differential equations.

\section{References}

[1] B. Abbo, Nouvel algorithme numérique de résolution des equations différentielles ordinaires (EDO) et des equations aux dérivées partielles (EDP) non linéaires, Thèse de doctorat unique, Université de Ouagadougou, Janvier 2007, UFR/SEA; Département de Mathématiques et Informatique, Burkina Faso.

[2] Bakari Abbo, Ngarkodje Ngarasta, Benjamin Mampassi, Blaise Some and Longin Some, A new approach of the Adomian algorithm for solving nonlinear partial or ordinary differential equations, Far East Journal of Applied Mathematics 23(3) (2006), 299-312.

[3] Joseph Bonazebi-Yindoula, Youssouf Pare, Francis Bassono and Gabriel Bissanga, Solving a linear convection-diffusion problem of Cauchy kind by Laplace-Adomian method, Far East Journal of Mathematical Sciences 101(3) (2017), 517-527.

DOI: http://dx.doi.org/10.17654/MS101030517

[4] M. Sambath and K. Balachandran, Laplace Adomian decomposition method for solving a fish farm model, Nonautonomous Dynamical Systems 3(1) (2016), 104-111.

DOI: https://doi.org/10.1515/msds-2016-0006

[5] S. A. Khuri, A Laplace decomposition algorithm applied to a class of nonlinear differential equations, Journal of Applied Mathematics 1(4) (2001), 141-155; Article ID 313078 .

DOI: https://doi.org/10.1155/S1110757X01000183

[6] M. Y. Ongun, The Laplace Adomian decomposition method for solving a model for HIV infection of $\mathrm{CD}^{+}{ }^{\mathrm{T}}$ cells, Mathematical and Computer Modelling 53(5-6) (2011), 597-603.

DOI: https://doi.org/10.1016/j.mcm.2010.09.009

[7] A. M. Wazwaz, The combined Laplace transform-Adomian decomposition method for handling nonlinear Volterra integro-differential equations, Applied Mathematics and Computation 216(4) (2010), 1304-1309.

DOI: https://doi.org/10.1016/j.amc.2010.02.023 
[8] M. Sh. Bani Issa, Ahmed A. Hamoud, Kirtiwant P. Ghadle and Giniswamy, Hybrid method for solving nonlinear Volterra-Fredholm integro differential equations, Journal of Mathematical and Computational Science 7(4) (2017), 625-641.

[9] M. Al-Mazmumy and H. Al-Malki, The modified Adomian decomposition method for solving nonlinear coupled Burger's equations, Nonlinear Analysis and Differential Equations 3(3) (2015), 111-122.

DOI: http://dx.doi.org/10.12988/nade.2015.41226

[10] K. Abbaoui and Y. Cherruault, The decomposition method applied to the Cauchy problem, Kybernetes 28(1) (1999), 68-74.

DOI: https://doi.org/10.1108/03684929910253261

[11] K. Abbaoui and Y. Cherruault, Convergence of Adomian's method applied to differential equation, Computers \& Mathematics with Applications 28(5) (1994), 103-109.

DOI: https://doi.org/10.1016/0898-1221(94)00144-8

[12] K. Abbaoui, Les Fondements de la Méthode Décompositionnelle d'Adomian et Application à la Résolution de Problèmes Issus de la Biologie et de la Medicine, Thèse de doctorat de l'Université Paris VI, October, 1995.

[13] K. Abbaoui and Y. Cherruault, Convergence of Adomian's method applied to nonlinear equations, Mathematical and Computer Modelling 20(9) (1994), 60-73.

DOI: https://doi.org/10.1016/0895-7177(94)00163-4

[14] T. Kato, On nonlinear Schrödinger equations, Annales de l'I.H.P. Physique Théorique 46(1) (1987), 113-129.

[15] Anne de Bouard, Equations Dispersives non Linéaires, Cours à l'Ecole d'Eté e de Mathématiques, Institut Fourier, Grenoble 20 Juin-8 Juillet, 2005.

[16] Travaux de thèse de doctorat unique du doctorant NKAYA Gires Dimitri, Faculté des Sciences et Techniques, Université Marien NGOUABI, Brazzaville-Congo, 2017.

[17] Syed Tauseef Mohyud-Din and Muhammad Aslam Noor, Homotopy perturbation method for solving partial differential equations, Zeitschrift für Naturforschung 64(a) (2009), 157-170.

[18] Blaise Some, Méthode Décompositionnelle d'Adomian et Méthode SBA, Cours et Exercices d'Application, L. A. NI.BIO. Université de Ouagadougou.

[19] P. Pue-on, Laplace Adomian decomposition method for solving Newell-WhiteheadSegel equation, Applied Mathematical Sciences 7(132) (2013), 6593-6600.

DOI: http://dx.doi.org/10.12988/ams.2013.310603

[20] A. Ghorbani and J. S. Nadjafi, He's homotopy perturbation method for calculating Adomian polynomials, International Journal of Nonlinear Sciences and Numerical Simulation 8(2) (2007), 229-332.

DOI: https://doi.org/10.1515/IJNSNS.2007.8.2.229 